\title{
Eine Verallgemeinerung, die keine ist
}

\author{
Zotcho Kumanov
}

Ohne Zweifel verdienen die Ethik sowie die ethischen Aspekte der Wirtschaft eine theoretische aber auch praktische Analyse. Das um so mehr, weil die wirtschaftliche Entwicklung eines jeden Landes eng mit den ethischen Normen, Traditionen, mit den rechtlichen und sozialpolitischen Zuständen verbunden ist.

Ethik, menschliches Benehmen und Verhalten gehören $\mathrm{zu}$ den exponierten Werten im Westen aber auch im Osten Europas. Das erreichte Niveau des ökonomischen Lebens, die Art und Weise der gesellschaftlichen Verhältnisse dort und hier zeigen aber einen bedeutenden Unterschied.

Eben deshalb halten wir die Darstellung von Franz Rupert Hrubi: "Business Ethics in Central and East Europe" für sehr aktuell:

- erstens, weil Wirtschaftsethik in Zentral und Osteuropa - im theoretischen sowie im praktischen Hinblick eine erst auf die Tagesordnung kommende Größe ist;

- zweitens, weil die Wirtschaftsethik im täglichen Geschäft und in der wirtschaftlichen Zusammenarbeit unterschiedlich bewertet wird;

- drittens, weil keine Einbahnstraße, weder westliche noch östliche Auffassungen von Wirtschaftsethik vorherrschend sein kann.

Das Interesse an der Wirtschaftsethik in den Ländern Zentral- und Osteuropas, wie der Autor der obigen Untersuchung meint, kommt nicht nur als ein Einfluß der Westeuropäer zustande, sondern auch als eine bewußte Suche nach Lösungen der ethisch-moralischen Probleme in allen Sektoren des Lebens. Man darf sogar behaupten, daß die zugespitzte ethische Problematik der Reformländer eine gewisse stimulierende Rolle für die verstärkte Aufmerksamkeit für wirtschaftsethische Fragen im Westen spielt.

Neben einigen frischen Feststellungen wie: "die westliche Ökonomie braucht mehr Ethik, ohne dabei das System zu verändern", oder "fast ein Jahrzehnt befinden sich die Zentral- und Osteuropäische Länder in einem Transformationsprozeß", wobei "die einzelnen Länder unterschiedlichen Fortschritt erzielt haben", lesen wir weiter bei Hrubi recht simple, vereinfachte Deutungen des Zustandes im Bereich der Business Ethik. Meiner Meinung nach geschieht das ohne ausreichende Argumente. So schreibt Hrubi z.B.: "Nach dem zweiten Weltkrieg war die Mehrheit dieser Länder vom Kommunismus erobert" oder "keiner hatte die Menschen gefragt, ob sie den Kommunismus billigen". 
Als ob nun jemand dieselben Länder fragte, ob sie den Kapitalismus billigen. Bloße Erklärungen bleiben die verbale Kritiken des Marxismus, Lenins und Stalins. Das ist Vergangenheit, mit der kann nicht die Gegenwart begründet werden. Schuldzuweisungen sind meiner Meinung nach keine wissenschaftliche Betrachtung, denn heutzutage fragt ja auch keiner nach der Ethik der Inquisition im Mittelhalter oder während des Krieges.

Was nützt nun die Behauptung von Hrubi: “jeder weiß, daß Marx leidenschaftlich böse Worte sprach, weil er dachte, daß die herrschende Moral, die Moral der herrschenden Klasse ist". Marx und der Marxismus sind bereits Jahrzehnte lang kritisiert worden. Die Doppelmoral, die Gesellschaft mit doppeltem Boden /Hrubi/ gab und es gibt immer noch ausnahmslos. Nicht ausschließlich im sogenannten Ostblock. Ebenso finden wir sie in der Logik der profitorientierten Wirtschaft, also dort wo der Mensch und seine ethische Werte in den Schatten des Gewinns gestellt worden sind.

Politisch-ideologische Besessenheit in der Art: “40, 70 Jahre unter der Diktatur des Proletariats haben dazu geführt, daß die Menschen mißtrauisch geworden sind, daß viele ihre moralische Ehre verloren haben, daß alle gezwungen waren, jemandem zu helfen", erinnert an eine sinnlose und veraltete Ausdrucksweise, die weder die gegenwärtige Debatte zu Wirtschaftsethik begreiflich macht, noch irgendeine brauchbare These der Wirtschaftsethik präsentiert.

Ganz anders und völlig mit Recht schreibt Hrubi, wenn er die Gleise des bösen Mannes verläßt: "Jede langjährige Wirtschaftsreform fuhrt zu gefährlichen, instabilen Zustände: die Preise wachsen, die Gehälter fallen, die Arbeitslosigkeit steigt" usw.

Solche Bilder kennen wir in allen Ländern Zentral- und Osteuropas. Eben deshalb sollen - auch im ethischen Sinne - die Ursachen, die Folgen und die Mechanismen der katastrophalen Lage der Menschen erforscht werden. Selbst die Neuen Länder in der Bundesrepublik leiden unter der Last der Wende. Und nicht vor allem wie Hrubi sagt, weil die Intellektuellen in unseren Ländern klarer als die Kollegen im Westen, die Untauglichkeit des Kommunismus und des Kapitalismus sehen, sondern weil die Menschen tagtäglich der Multi-Ethik des Lebens begegnen.

Wir plädieren für eine De-Ideologisierung der Ethik, für eine Sozialisierung der Wirtschaft und keine Trennung von Wirtschaftsethik in Ost und West. Am Beispiel des heutigen Europas beobachten wir eine kurzsichtige, ja seltsame Ethik. Die EU hat neue Grenzen, egal wie sie begründet werden. Daß sich innerhalb dieser durch das Schengener Abkommen festgelegten Grenzen eine eigene Wirtschaftsethik entwickeln wird, wird als Versuch gewertet, ganze Länderregionen wirtschaftlich abzukoppeln; was eigentlich keine Denkweise im kontinentalen Rahmen ist. In diesem Sinne ist eine Verallgemeinerung, die Wirtschaftsethik Zentral- und Ost Europas zu begründen, äußerst schwer. Man 
sollte die Länder gut kennen, denn dort wo gutes Bier gebraut wird, hat man ja nicht unbedingt Ahnung von gutem Wein. Es wäre Träumerei, induktiv Schlüsse zu ziehen, die ein Mosaiksteinchen im Rahmen des vereinten Europas darstellen. Hüten sollten wir uns vor zu leichtfertigen Prädikaten, unter Umständen besonders dort, wo die Marktwirtschaft noch in den Kinderschuhen steckt und die Ethik nur als eine Widerspiegelung des Überlebens Geltung hat.

Es gibt genug Anzeichen dafür, daß wir alle uns in einer Trasnformation hin zu einer globalisierten Welt befinden. Wenn $\mathrm{zu}$ Wirtschaftsethik geforscht und darüber diskutiert wird, sollten wir dann nicht die Frage über die monopolisierende Ethik stellen? Monopolisierend durch Kommunikation, Technologien, überregionale Macht- und Produzentenstrukturen. Unter diesem Blickwinkel, erscheint Wirtschaftsethik keine spezifische Zentral- und osteuropäische Frage zu sein, sondern eine gesamteuropäische. Jeder Versuch, Ethik Modelle unter schwachen Wirtschaften zu entwickeln ist zum scheitern verurteilt, denn es ist unmöglich, mit solchen Modellen z.B. Bulgarien, wo pro Stunde 2 DM bezahlt werden, mit Deutschland, einem Land wo pro Stunde 40 DM bezahlt werden, zu vergleichen.

Hrubi sollte diese simple Differenz, die eigentlich für alle Länder Zentral - und Osteuropas gültig ist, sehen. Eben vom Dasein beginnt das Bewußtsein, an diesem Grenzpunkt treffen sich und trennen sich Wirtschaft und Ethik.

Neben allen anderen Ethikbegriffen, gibt es auch die Ethik der Wissenschaft. Eine brennende Aufgabe wäre nicht nur die Wichtigkeit der Ethik für die Wirtschaft nachzuweisen, sondern den Mut zu haben und zu ergründen, wie die Kluft zwischen Ethik des täglichen Lebens und der Wirtschaft zu überbrücken ist sowie die Mechanismen des Wirkens in einer kaputten, kaum atmenden, von Kriminalität und Korruption durchdrungenen Wirtschaft der Gesellschaft vor Augen zu führen. Also: Die Philosophen haben die Welt nur verschieden interpretiert, es kommt drauf an, sie zu verändern. Dies sollte Franz Hrubi auch gelesen haben, als er das Beweismaterial analysierte. 